UCRL- JC-121556

PREPRINT

\&.

\title{
Overview of the Current Spectroscopy Effort on the Livermore Electron Beam Ion Traps
}

\author{
P. Beiersdorfer \\ G. Brown \\ J. Crespo López-Urrutia \\ V. Decaux \\ S.R. Elliott \\ D. Savin \\ A.J. Smith \\ G. Stefanelli \\ K. Widmann \\ K.L. Wong
}

Prepared for Submittal to

Proceedings of the 1st Euroconference on Atomic Physics with Stored Highly Charged Ions Heidelberg, Germany, March 20-24, 1995

June 29, 1995

This is a preprint of a paper intended for publication in a journal or proceedings. Since changes may be made before publication, this preprint is made available with the understanding that it will not be cited or reproduced without the permission of the author. 


\section{DISCI.AIMER}

This document was prepared as an account of work sponsored by an agency of the United States Government. Neither the I'nited States Government nor the Iniversity of ('alifornia nor any of their employees. makes any warranty, express or implied, or assumes any legal liability or responsibility for the accuracy, completeness. or usefulness of any information, apparatus, product, or process disclosed, or represents that its use would not infringe privately owned rights. Reference herein to any specific commercial products, process, or service by trade name, trademark, manufacturer, or otherwise. does not necessarily constitute or imply its endorsement, recommendation. or favoring by the linited States Government or the University of California. The views and opinions of authors expressed herein do not necessarily state or reflect those of the linited States Garernment or the lniversity of California, and shall not be used for advertising or praduct endorsement purposes. 


\section{DISCLAIMER}

Portions of this document may be illegible in electronic image products. Images are produced from the best available original document. 


\title{
Overview of the current spectroscopy effort on the Livermore Electron Beam Ion Traps
}

\author{
P. Beiersdorfer, ${ }^{1}$ G. Brown, ${ }^{2}$ J. Crespo López-Urrutia ${ }^{1}$ V. Decaux, ${ }^{1}$ S. R. Elliott ${ }^{1 \#}$ \\ D. Savin, ${ }^{3}$ A. J. Smith, ${ }^{4}$ G. Stefanelli, ${ }^{5}$ K. Widmann, ${ }^{1}$ K. L. Wong 1 \\ 1 Lawrence Livermore National Laboratory, Livermore, CA 94550 \\ ${ }^{2}$ Auburn University, Auburn, AL, 36849 \\ ${ }^{3}$ University of California, Berkeley, CA 94720 \\ ${ }^{4}$ Morehouse College, Atlanta, GA 30314 \\ 5 University of Nevada, Reno, NV 89557
}

An overview is given of the current spectroscopic effort on the Livermore electron beam ion trap facilities. The effort focuses on four aspects: spectral line position, line intensity, temporal evolution, and line shape. Examples of line position measurements include studies of the $\mathrm{K}$-shell transitions in heliumlike $\mathrm{Kr}^{34+}$ and the $2 \mathrm{~s}-2 \mathrm{p}$ intrashell transitions in lithiumlike $\mathrm{Th}^{87+}$ and $\mathrm{U}^{89+}$, which provide benchmark values for testing the theory of relativistic and quantum electrodynamical contributions in high- $Z$ ions. Examples of line intensity measurements are provided by measurements of the electron-impact excitation and dielectronic recombination cross sections of heliumlike transition-metal ions $\mathrm{Ti}^{20+}$ through $\mathrm{Co}^{25+}$. A discussion of radiative lifetime measurements of metastable levels in heliumlike ions is given to illustrate our time-resolved spectroscopy techniques in the microsecond range. We also present a measurement of the spectral lineshape that illustrates the very low ion temperatures that can be achieved in an EBIT.

\#present address: Nuclear Physics Laboratory, GL-10, University of Washington, Seattle, WA 98195 


\section{Introduction}

The electron beam ion trap (EBIT) was designed for the spectroscopic study of atomic processes of highly charged ions [1]. The device provides direct line-of-sight access to the region were an electron beam produces and excites highly charged ions of a particular species of interest, thus allowing for detailed studies of electron-ion interactions with highresolution spectroscopic means. This direct spectroscopic access and the fact that the highly charged ions are virtually at rest in the trap makes EBIT unique among the present generation of atomic physics facilities, such as heavy-ion storage rings, electron beam ion sources (EBIS), and electron cyclotron resonance (ECR) sources. The low-energy version of EBIT employs an electron beam with energy as high as $30 \mathrm{keV}$ and allows for the study of bare molybdenum. A high-energy version, dubbed SuperEBIT, was recently completed [2]. It employs electron beams with energies as high as $200 \mathrm{keV}$ to produce fully stripped uranium ions [3].

Because of the high charge states involved most spectroscopic efforts have been concentrated on analyzing radiation in the $\mathrm{x}$-ray regime [4]. A large number of measurements have been carried out spanning a wide range of ionization stages around the closed-shell heliumlike, neonlike, and nickellike systems [5]. A variety of high-resolution flat-crystal and curved-crystal spectrometers are available for this purpose [6,7] providing the necessary degree of flexibility in matching resolving power and throughput with the requirements of a given measurement. The range of x-ray energies spanned by these instrument presently extends from 650 to $15,000 \mathrm{eV}$. The high-resolution crystal spectrometers are complemented with a pulse-height analysis system utilizing high-purity germanium or $\mathrm{Si}(\mathrm{Li})$ detectors and providing a broad-band analysis of the $\mathrm{x}$-ray emission from EBIT from 0.5 to $350 \mathrm{keV}$. Highly charged ions, however, also radiate profusely at energies below that of the $\mathrm{x}$-ray regime. This is illustrated in a recent discovery of optical transitions from highly charged xenon ions studied at the EBIT facility at the National Institute of Standards and Technology [8]. We use a set of high-resolution prism and transmission-grating spectrometers at Livermore to study optical transitions in the 
wavelength band from 3700 to $6900 \AA$. Forbidden transitions of diagnostic interest are currently under study.

Spectroscopy on the EBIT facility provides atomic physics information that can be divided into four general categories:

(1) Position of the spectral lines. Crucial for the precise determination of the energy levels, for line identification and line blends, as well as tests of relativity and quantum electrodynamics.

(2) Intensity of the spectral lines. It allows the measurement of the electron-ion interaction cross sections. The cross section measurements include electron-impact excitation, dielectronic recombination, innershell ionization, and resonance excitation. The intensity information also allows the study of polarization phenomena.

(3) The temporal evolution of the spectral lines. It allows the study of transient phenomena such as innershell ionization and line emission by radiative electron capture. It also allows the study of radiative lifetimes of long-lived excited states.

(4) Line shape information. It allows a determination of the temperature of the trapped ions from the thermal Doppler broadening and thus provides basic information on the trap performance.

The electron beam energy provides yet another dimension to the range of experimental conditions EBIT can access. The beam is quasi mono-energetic with an energy spread of 30-50 eV FWHM and can be changed at a rate of $50 \mathrm{~V} / \mathrm{ns}$, i.e., at a rate faster than that of the electron-ion interaction process. As a result, we can use EBIT to make an ion at one energy and probe it with electrons at a second energy, selecting the electron-ion interaction process of interest. The line formation processes in EBIT are thus very different from those in a plasma, where virtually all processes occur simultaneously.

Our current spectroscopic effort touches on each of the four categories listed above. In the following, we present specific examples that illustrate the current activities in each of the categories. These include the precise measurements of the $n=2$ to $n=1$ transitions in heliumlike $\mathrm{Kr}^{34+}$ and of $n=2$ to $n=2$ intrashell transitions in lithiumlike thorium and 
uranium ions, which provide experimental benchmarks for testing relativistic and QED calculations; measurements of the electron-impact excitation and dielectronic recombination cross sections of heliumlike transition-metal ions determined from the observed intensities; a discussion of radiative lifetime measurements; and measurements of spectral lineshapes to illustrate the very low ion temperatures that can be achieved in an EBIT. Additional examples of our current spectroscopic effort are discussed by $D$. Schneider and Th. Stöhlker in this volume.

\section{K-shell transition energies of heliumlike $\mathbf{K r}^{34+}$}

Spectra of heliumlike ions have been a subject of intense research interest, as these ions provide an ideal setting for testing approaches to solve the many-body problem. Several theoretical predictions of the energy levels have been published recently that employ different ways to account for electron correlations and the many-body quantum electrodynamical corrections $[9,10]$. While the calculations tend to agree with each other for low- $Z$ heliumlike ions, significant differences exist among the predictions for high- $Z$ heliumlike ions. Experimental results are necessary in order to distinguish among the various theoretical approaches and to guide their further development.

Heliumlike $\mathrm{K} \alpha$ radiation has been studied for ions as heavy as $\mathrm{Xe}^{52+}$ and $\mathrm{U}^{90+}$ [11] but only up to $\mathrm{Kr}^{34+}$ with high-resolution crystal spectrometers [12]. The highest accuracy for the $\mathrm{Kr}^{34+} \mathrm{K} \alpha$ transition energies was achieved by Indelicato et al. [12] at the GANIL accelerator facility ( $24 \mathrm{ppm})$ but the experimental results differ significantly from all recent calculations. There is a strong interest in remeasuring the energies of the $\mathrm{K} \alpha$ transitions of heliumlike krypton to either validate or invalidate the previous results. Using the LLNL EBIT facility we have made such a measurement attaining a precision ranging between 20 and $33 \mathrm{ppm}$ for the different transitions measured.

Our measurement was performed using a von Hámos-type high-resolution crystal spectrometer. This setup measures the $\mathrm{Kr} \mathrm{K}$-shell radiation in second order and the hydrogenic $\mathrm{Mn}^{24+}$ and heliumlike $\mathrm{Fe}^{24+}$ spectra used for calibration in first order Bragg 
reflection as shown in Fig. 1. The energies of four $\mathrm{Kr}^{34+}$ transitions were measured. The set includes the ${ }^{1} \mathrm{P}_{1}$ and ${ }^{3} \mathrm{P}_{1}$ transitions measured earlier at GANL [12], as well as the ${ }^{3} \mathrm{P}_{2}$ and ${ }^{3} S_{1}$ transitions shown in Fig. 1, which had not been measured before.

The uncertainty in the determination of the transition energies results from the uncertainty of the calibration lines, the uncertainty in the determination of the centroid of each line, and the uncertainty of the dispersion along the detector. The accuracy of our measurements was limited mainly by the uncertainty of the iron calibration lines, which for the different lines ranges from 43 to $59 \mathrm{ppm}$.

Our measurements are in excellent agreement with most recent calculations [13]. Best agreement is found with the theory of Cheng et al. [10], where the average difference between our measured values and their predictions is $0.05 \pm 0.36 \mathrm{eV}$. By contrast, the earlier measurements at the GANIL accelerator facility [12] disagree with their predictions by $0.60 \pm 0.30 \mathrm{eV}$.

\section{Lamb shift measurements of lithiumlike $\mathrm{Th}^{87+}$ and $\mathrm{U}^{89+}$}

Energy shifts due to quantum electrodynamical effects increase as $\mathrm{Z}^{4}$. The QED-induced energy shift of the $2 s_{1 / 2}$ level in lithiumlike $\mathrm{U}^{89+}$ and lithiumlike $\mathrm{Th}^{87+}$ is more than 40 $\mathrm{eV}$. This shift represents a large fraction of the overall $2 s_{1 / 2}-2 p_{1 / 2}$ or $2 s_{1 / 2}-2 p_{3 / 2}$ transition energies, and a precise measurement of these transitions is a sensitive test of the predictions of QED theory. Using SuperEBIT and a high-resolution crystal spectrometer we have made a measurement of thirteen $2 \mathrm{~s}_{1 / 2}-2 \mathrm{p}_{3 / 2}$ transitions in the eight ionization states $\mathrm{Th}^{80+}$ through $\mathrm{Th}^{87+}[14]$ as well as in the eight ionization states $\mathrm{U}^{82+}$ through $\mathrm{U}^{89+}$ [15]. The measurements achieved an accuracy between $0.15 \mathrm{eV}$ and $0.45 \mathrm{eV}$.

A high-resolution spectrum of the $2 \mathrm{~s}_{1 / 2}-2 \mathrm{p}_{3 / 2}$ transitions in lithiumlike $\mathrm{Th}^{87+}$ through oxygenlike $\mathrm{Th}^{82+}$ is shown in Fig. 2 . In recording the $2 \mathrm{~s}_{1 / 2}-2 \mathrm{p}_{3 / 2}$ transitions the ionization balance in the trap was tuned by changing the electron energy, the neutral gas density (i.e., the charge-exchange recombination rate), and the effective current density, in 
order to study of the full range of charge states from neonlike to lithiumlike. From these measurements we determined $4459.37 \pm 0.21 \mathrm{eV}$ for the $2 \mathrm{~s}_{1 / 2}-2 \mathrm{p} 3 / 2$ transitions in lithiumlike $U^{89+}$ with a $1-\sigma$ confidence level [15]. The energy of the $2 s_{1 / 2}-2 p_{3 / 2}$ transition in lithiumlike $\mathrm{Th}^{87+}$ was determined to be $4025.23 \pm 0.14 \mathrm{eV}$ [14]. These results are in excellent agreement with recent $a b$ initio calculations [16] of the QED energies. The agreement with theory is illustrated in Fig. 3, where we have plotted the fractional difference between measured and calculated QED contributions to the $2 s_{1 / 2}-2 p_{3} / 2$ transitions in lithiumlike ions.

Our measurements of the $2 \mathrm{~s}_{12}-2 \mathrm{p}_{3 / 2}$ transitions complement the earlier experiment of Schweppe et al. who measured the energy of the $2 \mathrm{~s}_{1 / 2}-2 \mathrm{p}_{1 / 2}$ transition in lithiumlike $\mathrm{U}^{89+}$ and found $280.59 \pm 0.10 \mathrm{eV}$ [17]. Their measurement together with our two measurements represent the best tests of QED in the limit $(Z \alpha) \approx 1$, where all-order calculations are imperative. The measurements represent a check of QED contributions to the $2 \mathrm{~s}-2 \mathrm{p}$ transitions (ranging from 36 to $48 \mathrm{eV}$ ) at the sub-1-\% level. This accuracy is nearly two orders of magnitude higher that achieved in measurements of the $L y-\alpha$ transitions of $\mathrm{U}^{91+}$, where the 270-eV QED contribution has been measured with an accuracy of tens of eV [18].

\section{Electron-impact excitation cross sections}

The intensity of a given line represents a direct measure of the excitation cross sections. To determine the cross section, we normalize the line intensity to the intensity of the photons emitted in the radiative electron capture. The cross sections for the latter process are reliably known, especially for the high electron energies of the EBIT measurements, and thus provide an ideal reference for normalization. This method was used in an earlier measurement of the electron-impact excitation cross sections of heliumlike $\mathrm{Ti}^{20+}$ [19]. Here we studied the excitation cross sections of the forbidden line $z$ ( $\left.1 \mathrm{~s} 2 \mathrm{~s}{ }^{3} \mathrm{~S}_{1}\right)$, intercombination lines $y\left(1 \mathrm{~s} 2 \mathrm{p}{ }^{3} \mathrm{P}_{1}\right)$ and $x\left(1 \mathrm{~s} 2 \mathrm{p}{ }^{3} \mathrm{P}_{2}\right)$, and resonance line $w\left(1 \mathrm{~s} 2 \mathrm{p}{ }^{1} \mathrm{P}_{1}\right)$. Recently, we have applied the technique to extend our measurement the electron-impact excitation cross sections along the isoelectronic sequence [20]. The ions studied were 
heliumlike $\mathrm{Ti}, \mathrm{V}, \mathrm{Cr}, \mathrm{Mn}$, and $\mathrm{Fe}(\mathrm{Z}=22-26)$. Results are shown in Fig. 4. The measurements were made at approximately $100 \mathrm{eV}$ above the excitation threshold for line $w$ where no processes other than direct excitation can contribute to the formation of the lines.

The uncertainties in the measurements are less than $8 \%$ for the heliumlike Fe transitions. These need to be combined with a $3 \%$ systematic uncertainty from the normalization to theoretical radiative recombination cross sections. We compared our measurements to results from a distorted-wave calculation and found good agreement. Very recently, closecoupling calculations have become available for the excitation of these ions [21]. Again, very good agreement with our data is found.

\section{Dielectronic recombination in $\mathrm{Co}^{25+}$}

Dielectronic recombination (DR) is the dominant recombination process in low density plasmas such as those found in the sun's corona and in tokamak fusion devices. It is a resonance process in which a highly charged ion captures an electron and simultaneously excites a bound electron to an excited state. Such a doubly-excited autoionizing state may be stabilized by the emission of a photon (dielectronic recombination); or it may undergo autoionization. Using similar techniques as outlined above for the determination of electron-impact excitation cross sections, we have studied line formation by dielectronic recombination in heliumlike $\mathrm{Co}^{25+}[22]$.

In the KLL process studied, a heliumlike ion captures an electron into the $L$ shell and excites an electron from the $\mathrm{K}$ to the $\mathrm{L}$ shell:

$$
1 s^{2}+e \rightarrow 1 s 212 l^{\prime} \rightarrow 1 s^{2} 2 \mathrm{l}+\mathrm{h} v^{\prime}
$$

The stabilizing transitions generate lithiumlike satellites to the heliumlike $\mathrm{K} \alpha$ lines. The emitted photon energies $h v^{t}$ of the satellite transitions are close to the energies $h v$ of the heliumlike transitions, 


$$
1 \mathrm{~s} 2 \mathrm{l} \rightarrow 1 \mathrm{~s}^{2}+\mathrm{hv}
$$

since the effect of the spectator electron 21 is rather small. Similarly, recombinations into the lithiumlike ground state with $\mathrm{K}$-shell excitation lead to berylliumlike satellites of the heliumlike $\mathrm{K} \alpha$ lines.

By sweeping the electron beam energy across the individual dielectronic recombination resonances, we have determined the relative resonance strengths of the strongest resonances populating doubly excited levels in lithiumlike and berylliumlike cobalt [22]. A spectrum showing the $\mathrm{x}$-ray lines produced by sweeping over all KLL resonances is shown in Fig. 5. Here, stabilizing $x$ rays from resonances in heliumlike $\mathrm{Co}^{25+}$ are labeled with lower case letter, $\mathrm{x}$ rays from resonances in lithiumlike $\mathrm{Co}^{24+}$ are labeled with numerals. Also shown are two theoretical spectra for comparison.

Most measured dielectronic satellite strengths compare well with theory. This is true especially for the strongest resonances that dominate the total recombination rate. A discrepancy with theoretical values is noted only for the weak resonances. These include the resonance strengths associated with levels $\left(1 \mathrm{~s}_{1 / 2} 2 \mathrm{p}^{2} 3 / 2\right)_{3 / 2}$ and $\left(1 \mathrm{~s}_{1 / 2} 2 \mathrm{~s}_{1 / 2} 2 \mathrm{p}_{1 / 2}\right)_{1 / 2}$. Their values of (49.8 \pm 3.2$) \times 10^{-20} \mathrm{~cm}^{2} \mathrm{eV}$ and $(22.9 \pm 6.4) \times 10^{-20} \mathrm{~cm}^{2} \mathrm{eV}$, respectively, are almost twice the size predicted by theory. Similar observations were made earlier in the case of iron, i.e., the neighboring element [23].

\section{Radiative lifetime of the $1 s^{2} s^{3} S_{1}$ level in heliumlike ions}

Given a set of basis functions, radiative transition rates for the electric-dipole allowed transitions are calculated from the dipole matrix elements. Radiative transition rates, therefore, provide information on the long-range behavior of atomic wavefunctions. Measurements of radiative transition rates are thus complementary to energy level measurements and test atomic structure calculations in a different and complementary way. Calculations of the radiative decay rates of dipole-forbidden transitions are highly sensitive to the theoretical approaches used and thus theoretically the most uncertain. As a result, 
measurements of the lifetimes of metastable levels are of particular interest. Moreover, the radiative lifetime of the metastable $1 \mathrm{~s} 2 \mathrm{~s}{ }^{3} \mathrm{~S}_{1}$ level in heliumlike ions determines the range over which the $\mathrm{K}$-shell emission lines from heliumlike ions can be used as electron density diagnostics, as illustrated in Fig. 6 . Accurate knowledge of the lifetime of the $1 \mathrm{~s} 2 \mathrm{~s}{ }^{3} \mathrm{~S}_{1}$ level is thus of great importance to plasma diagnostics, especially of solar and astrophysical plasmas.

Recently, we have developed a technique for measuring radiative lifetimes in highly charged heliumlike ions [24]. The technique relies on rapid switching of the energy of the electron beam, above and below the excitation threshold of the ${ }^{3} S_{1}$ level, and determining the lifetime of the metastable ${ }^{3} \mathrm{~S}_{1}$ level from its fluorescent decay while the beam energy was below threshold. The new technique can be used over eight orders of magnitude in time, from a few nsec to hundreds of msec. Most of this region is inaccessible to any other existing method such as accelerator beam-foil measurements, laser fluorescence techniques, or ion traps combined with ECR sources, as illustrated in Fig. 6. The EBIT measurements are also complementary to the lifetime measurements that have been performed with novel techniques on ion storage rings [25]. The EBIT technique enables measurements of radiative lifetimes in heliumlike ions that are useful density diagnostics in the range $10^{8} \mathrm{~cm}^{-3}$ to $10^{15} \mathrm{~cm}^{-3}$, as illustrated in Fig. 6. Adding to our previous measurement of the $(90.5 \pm 1.5)$ - $\mu$ s radiative lifetime of the ${ }^{3} \mathrm{~S}_{1}$ level in heliumlike $\mathrm{Ne}^{8+}$ [24] we have recently determined the (13.6 \pm 0.5$)$ - $\mu$ s radiative lifetime of the ${ }^{3} S_{1}$ level in heliumlike $\mathrm{Mg}^{10+}$ [26], which is used as a diagnostic in plasmas with electron densities near $10^{12} \mathrm{~cm}^{-3}$.

\section{Production of cold highly charged ions}

The characteristic $\mathrm{x}$-ray lines of highly charged ions from virtually all plasma sources are broadened by the thermal motion of the emitting ions. The line emission from many plasmas, such as laser-produced plasmas, may be broadened further by opacity or density effects. The line emission observed in accelerator-based measurements is broadened by the Doppler effect, because the necessity for a finite acceptance angle entails viewing 
relativistic ions with different velocity components. The line emission from any of these sources may be broadened yet more by satellite lines populated by single or multiple electron capture into high-lying spectator levels. As a result of this broadening, the resolution and the precision with which measurements can be made is limited.

EBIT uses a monoenergetic electron beam to produce and excite highly charged ions in a trap. Because excitation processes can be selected by choosing the appropriate energy, the population of satellite transitions that may broaden a given line in plasma and accelerator sources is avoided. Moreover, the electron density is less than $5 \times 10^{12} \mathrm{~cm}^{-3}$ so that opacity or density effects are unimportant and broadening effects that may affect high-density plasma sources are absent. On the other hand, interaction with the electron beam causes strong heating of the ions [1]; thus ion thermal broadening is expected to play a role in determining the widths of $\mathrm{x}$-ray lines produced with an EBIT device. If a systematic approach can be found to further reduce the temperature of the ions produced and trapped in EBIT, a potentially large gain in spectroscopic precision could be achieved, as the precision with which transition energies can be measured is directly related to the line width.

We have begun to perform systematic studies to identify the parameters that affect the temperature of the ions produced and trapped in the EBIT device [27]. For this we note that the temperature of the ions is limited by the fact that ions with enough kinetic energy to overcome the trapping potential will leave the trap and are lost. By varying the trap depth, we were able to change the temperature from as high as $1600 \mathrm{eV}$ to well below 100 $\mathrm{eV}$. The reduction of the spectral line width of the transition $\left(1 \mathrm{~s} 2 \mathrm{p}^{2}\right)_{5 / 2} \rightarrow\left(1 \mathrm{~s}^{2} 2 \mathrm{p}\right)_{3 / 2}$ (labeled $j$ ) and the transition (1s2 $\left.\mathrm{p}^{2}\right)_{3 / 2} \rightarrow\left(1 \mathrm{~s}^{2} 2 \mathrm{p}\right)_{1 / 2}$ (labeled $k$ ) in lithiumlike $\mathrm{Ti}^{19+}$ as the ion temperature changes from $685 \mathrm{eV}$ to $70 \mathrm{eV}$ is seen in Fig. 7. The line widths shown are among the smallest observed for $\mathrm{x}$-ray transitions from highly charged ions and thus allow very precise energy determinations. Our measurements indicate that further reductions in the ion temperature are feasable. 


\section{Conclusion}

As the brief illustrations have shown, the spectroscopic effort on EBIT and SuperEBIT comprises a large field of scientific investigation. New techniques, such as visible spectroscopy or the production of cold highly charged ions, are constantly being developed and added to an already large repertoire of experimental possibilities.

This work was supported in part by the U.S. Department of Energy, Office of Basic Energy Sciences and by the National Aeronautics and Space Administration under grant No. NAGW-4185 and was performed at the Lawrence Livermore National Laboratory under the auspices of the U.S. Department of Energy under contract No. W-7405-ENG-48. 


\section{References}

[1] M. A. Levine, R. E. Marrs, J. R. Henderson, D. A. Knapp, and M. B. Schneider, Phys. Scr. T22, 157 (1988).

[2] D. A. Knapp, R. E. Marrs, S. R. Elliott, E. W. Magee, and R. Zasadzinski, Nucl. Instrum. Methods A334, 305 (1993).

[3] R. E. Marrs, S. R. Elliott, D. A. Knapp, Phys. Rev. Lett. 72, 4082 (1994).

[4] M. A. Levine, R. E. Marrs, J. N. Bardsley, P. Beiersdorfer, C. L. Bennet, M. H. Chen, T. Cowan, D. Dietrich, J. R. Henderson, D. A. Knapp, A. Osterheld, B. M. Penetrante, M. B. Schneider, and J. H. Scofield, Nucl. Instrum. Methods B43, 431 (1989); R. E. Marrs, Comments At. Mol. Phys. 27, 57 (1991); P. Beiersdorfer, Nucl. Instrum. Methods B56/57, 1144 (1991).

[5] P. Beiersdorfer, in VIth International Conference on the Physics of Highly Charged Ions, AIP Conference Proceedings No. 274, ed. by P. Richard, M. Stöckli, C. L. Cocke, and C. D. Lin (AIP, New York, 1993), p. 365; P. Beiersdorfer, in Atomic Physics 14, ed. by D. J. Wineland, C. E. Wieman, and S. J. Smith (AIP, New York, 1995), p. 116.

[6] P. Beiersdorfer, R. E. Marrs, J. R. Henderson, D. A. Knapp, M. A. Levine, D. B. Platt, M. B. Schneider, D. A. Vogel, and K. L. Wong, Rev. Sci. Instrum. 61, 2338 (1990)

[7] P. Beiersdorfer and B. J. Wargelin, Rev. Sci. Instrum. 65, 13 (1994).

[8] C. A. Morgan, F. G. Serpa, E. Takács, E. S. Meyer, J. D. Gillaspy, J. Sugar, J. R. Roberts, C. M. Brown, and U. Feldman, Phys. Rev. Lett. 74, 1716 (1995).

[9] G.W. Drake, Can. J. Phys. 66, 586 (1988); D.R. Plante, W.R. Johnson, and J. Sapirstein, Phys. Rev. A 49, 3519 (1994)

[10] K.T. Cheng, M.H. Chen, W.R. Johnson, and J. Sapirstein, Phys. Rev. A 50, 247 (1994).

[11] J.P. Briand, P. Indelicato, A. Simionovici, V. San Vicente, D. Liesen, and D. Dietrich, Europhys. Lett. 9, 225 (1989); J.P. Briand, P. Chevallier, P. Indelicato, K.P. Ziock, and D.D. Dietrich, Phys. Rev. Lett. 65, 2761 (1990). 
[12] P. Indelicato, J.P. Briand, M. Tavernier, and D. Liesen, Z. Phys. D -- Atoms, Molecules and Clusters 2, 249 (1986).

[13] K. Widmann, P. Beiersdorfer, V.Decaux, and M. Bitter, Phys. Rev. A (in press).

[14] P. Beiersdorfer, A. Osterheld, S. R. Elliott, M. H. Chen, D. Knapp, and K. Reed, Phys. Rev. A (submitted).

[15] P. Beiersdorfer, D. Knapp, R. E. Marrs, S. R. Elliott, and M. H. Chen, Phys. Rev. Lett. 71, 3939 (1993); P. Beiersdorfer, Nucl. Instrum. Methods (in press).

[16] S. A. Blundell, W. R. Johnson, and J. Sapirstein, Phys. Rev. A 41, 1698 (1990); S. A. Blundell, Phys. Rev. A 47, 1790 (1993).

[17] J. Schweppe, A. Belkacem, L. Blumenfeld, N. Claytor, B. Feinberg, H. Gould, V. E. Kostroun, L. Levy, S. Misawa, J. R. Mowat, and M. H. Prior, Phys. Rev. Lett. 66, 1434 (1991).

[18] J. P. Briand, P. Chevallier, P. Indelicato, K. P. Ziock, and D. Dietrich, Phys. Rev. Lett. 65, 2761 (1990); Th. Stöhlker, P. H. Mokler, K. Beckert, F. Bosch, H. Eickhoff, B. Franzke, M. Jung, T. Kandler, O. Klepper, C. Kozhuharov, R. Moshammer, F. Nolden, H. Reich, P. Rymuza, P. Spädtke, and M. Steck, Phys. Rev. Lett. 71, 2184 (1993); J. H. Lupton, D. D. Dietrich, C. J. Hailey, R. E. Stewart, and K. P. Ziock, Phys. Rev. A 50, 2150 (1994); H. Beyer, these proceedings.

[19] S. Chantrenne, P. Beiersdorfer, R. Cauble, and M. B. Schneider, Phys. Rev. Lett. 69, 265 (1992).

[20] K. Wong, P. Beiersdorfer, K. J. Reed, and D. A. Vogel, Phys. Rev. A 51, 1214 (1995).

[21] T. W. Gorczyca, F. Robicheaux, and M. S. Pindzola, Phys. Rev. A (submitted); H. L. Zhang and A. K. Pradhan, Phys. Rev. A (submitted).

[22] A. J. Smith, P. Beiersdorfer, V. Decaux, K. Widmann, A. Osterheld, and M. H. Chen, Phys. Rev. A 51, 2808 (1995).

[23] P. Beiersdorfer, T. W. Phillips, K. L. Wong, R. E. Marrs, and D. A. Vogel, Phys. Rev. A 46, 3812 (1992).

[24] B. J. Wargelin, P. Beiersdorfer, and S. M. Kahn, Phys. Rev. Lett. 71, 2196 (1993). 
[25] H. T. Schmidt, P. forck, M. Grieser, D. Habs, J. Kenntner, G. Miersch, R. Repnow, U. Schramm, T. Schüssler, D. Schwalm, and A. Wolf, , Phys. Rev. Rev. Lett. 72, 1616 (1994).

[26] G. S. Stefanelli, P.Beiersdorfer, V. Decaux, and K. Widmann, Phys. Rev. A (in press).

[27] P. Beiersdorfer, V. Decaux, S. R. Elliott, K. Widmann, and K. Wong, Rev. Sci. Instrum. 66, 303 (1995); P. Beiersdorfer, V. Decaux, and K. Widmann, Nucl. Instrum. Meth. (in press).

[28] L. A. Vainshtein and U. I. Safronova, At. Data Nucl. Data Tables 25, 49 (1978). 


\section{Figures}

Fig. 1. Bragg-crystal spectra of (a) hydrogenic $\mathrm{Mn}^{24+}$ showing the $\mathrm{Ly}-\alpha_{1,2}$ transitions and heliumlike $\mathrm{Fe}^{24+}$, showing the $1 \mathrm{~s} 2 \mathrm{~s}{ }^{3} \mathrm{~S}_{1} \rightarrow 1 \mathrm{~s}^{2}{ }^{1} \mathrm{~S}_{0}$ transition, labeled $z$; (b) heliumlike $\mathrm{Kr}^{34+}$ measured in second order. The $\mathrm{Kr}$ and $\mathrm{Fe}$ spectra also show collisional satellite lines of lower charge states. Three lithiumlike $(r, q, t)$ and one berylliumlike (B) satellite lines are labeled.

Fig. 2. Spectrum of $2 s_{1 / 2}-2 p_{3 / 2}$ electric dipole transitions in highly charged thorium. Lines are labeled by the charge state of the emitting ion; $\mathrm{Li}$, for example, denotes emission from lithiumlike $\mathrm{Th}^{87+}$.

Fig. 3. Difference between theory and experiment for the $2 s_{1 / 2}-2 p_{3 / 2}$ transition energy in the lithiumlike isoelectronic sequence expressed as a percentage of the theoretical QED energy (sum of self energy and vacuum polarization). The thorium and uranium points are from EBIT measurements; a listing of the references to the other experimental points is given in [16].

Fig. 4. Measurements of the effective electron-impact excitation cross sections of heliumlike ions (solid circles) for different elements for lines (a) $w$, (b) $x$, (c) $y$, and (d) $z$. The solid curve represents results from a relativistic distoted-wave calculation. Open triangles represent earlier measurements of the cross sections for heliumlike $\mathrm{Ti}^{20+}$.

Fig. 5. Dielectronic recombination satellite spectrum for heliumlike $\mathrm{Co}^{25+}$. experimental spectrum; (b) and (c) theoretical spectra from two different calculations, given in [22] and [28], respectively.

Fig. 6. Lifetime of the metastable $1 \mathrm{~s} 2 \mathrm{~s}{ }^{3} \mathrm{~S}_{1}$ level in heliumlike ions as a function of atomic number. The critical density at which the level is depopulated by electron collisions is given on the $y$-axis. The shaded areas indicate the regimes that can be 
tested with various techniques, such as the beam-foil, Penning-trap, and storage-ring method. The EBIT measurement of neon and magnesium are the first to test heliumlike lifetimes relevant for determinations of densities in stellar atmospheres.

Fig. 7. Crystal-spectrometer spectrum of the lines $j$ and $k$ from lithiumlike $\mathrm{Ti}^{19+}$ ions: (a) $685-\mathrm{eV}$ ion temperature and (b) $70-\mathrm{eV}$ ion temperature. The line widths reflect the thermal Doppler motion of the emitting ion. 

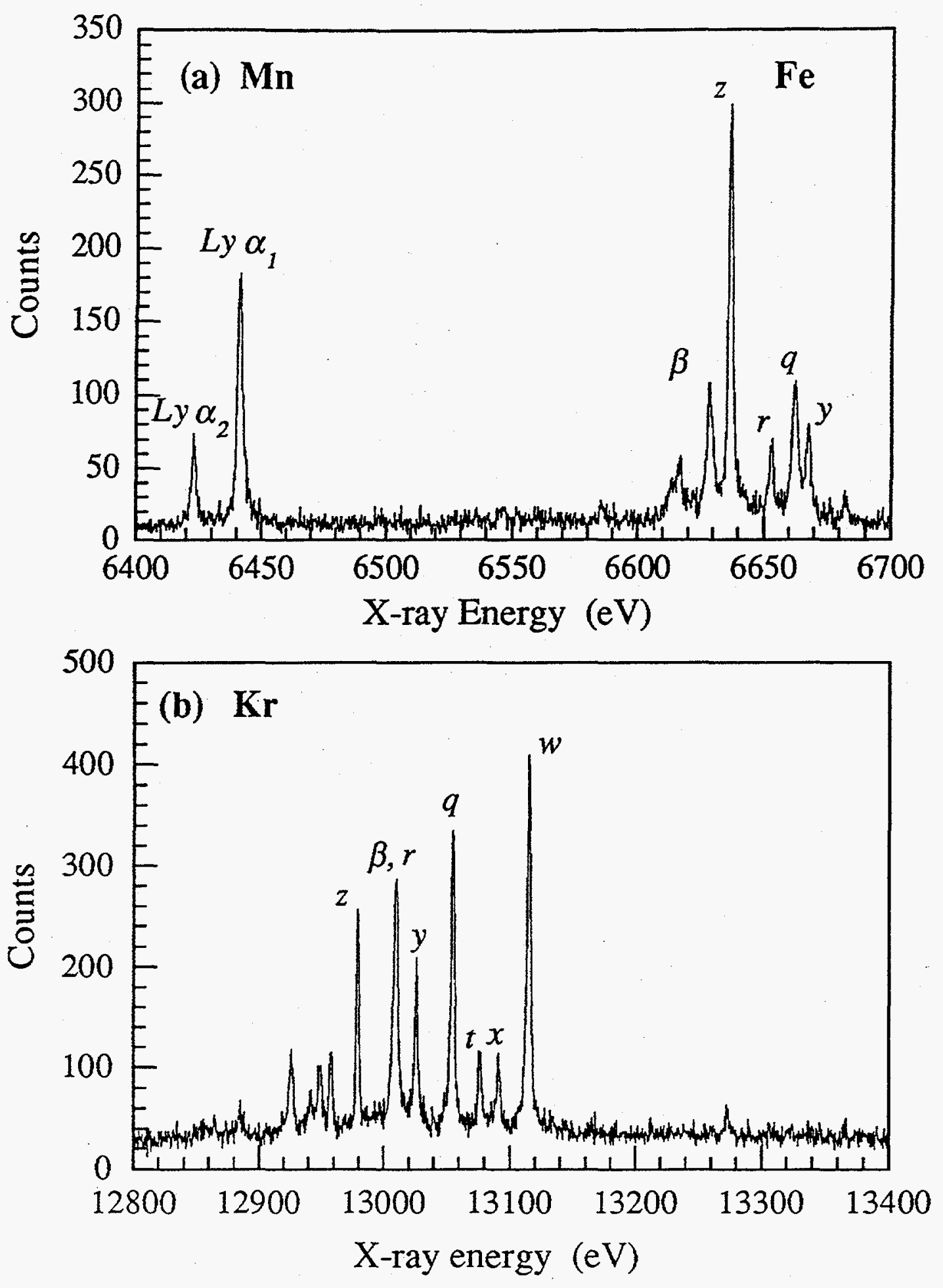

Figure 1. 


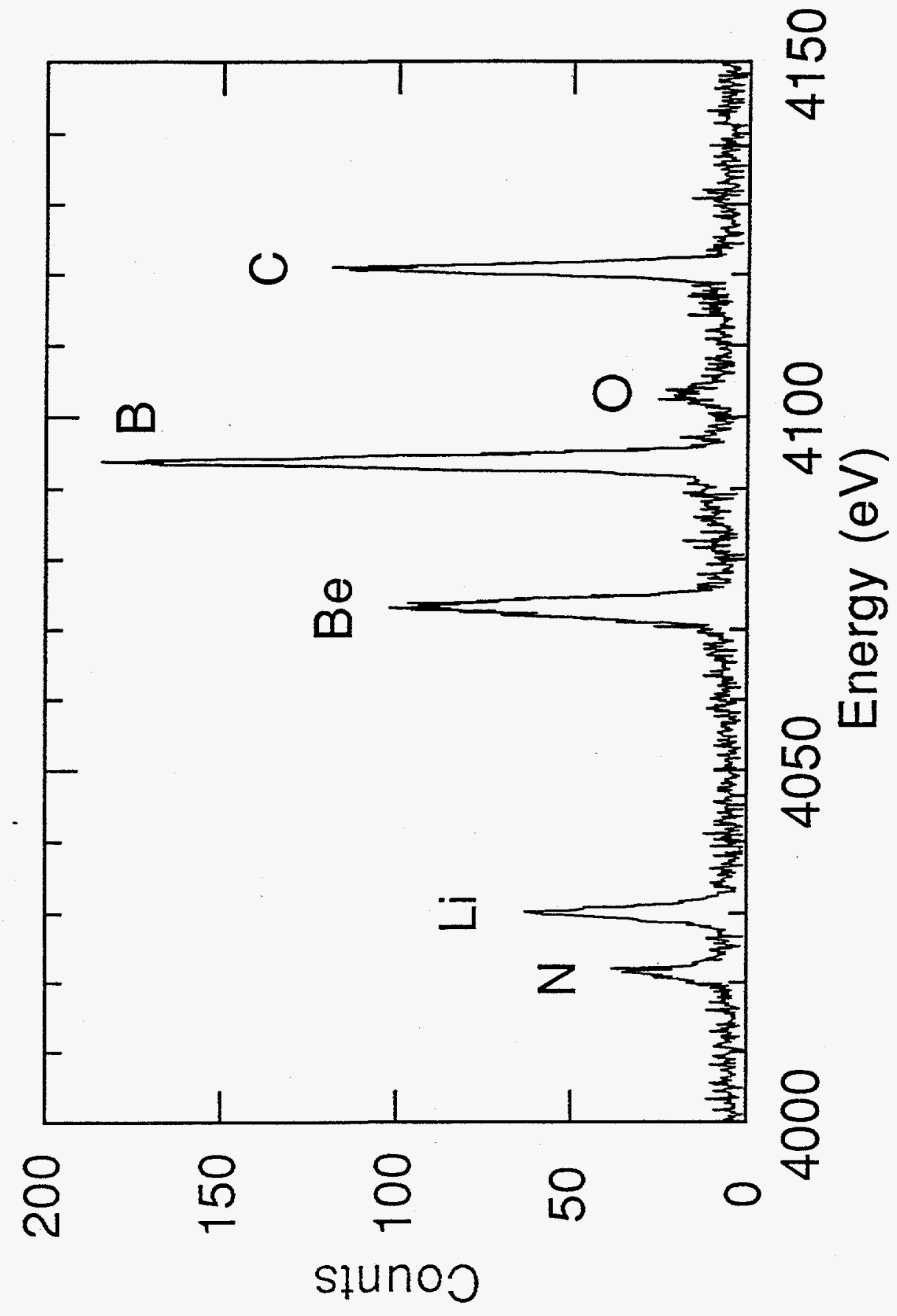




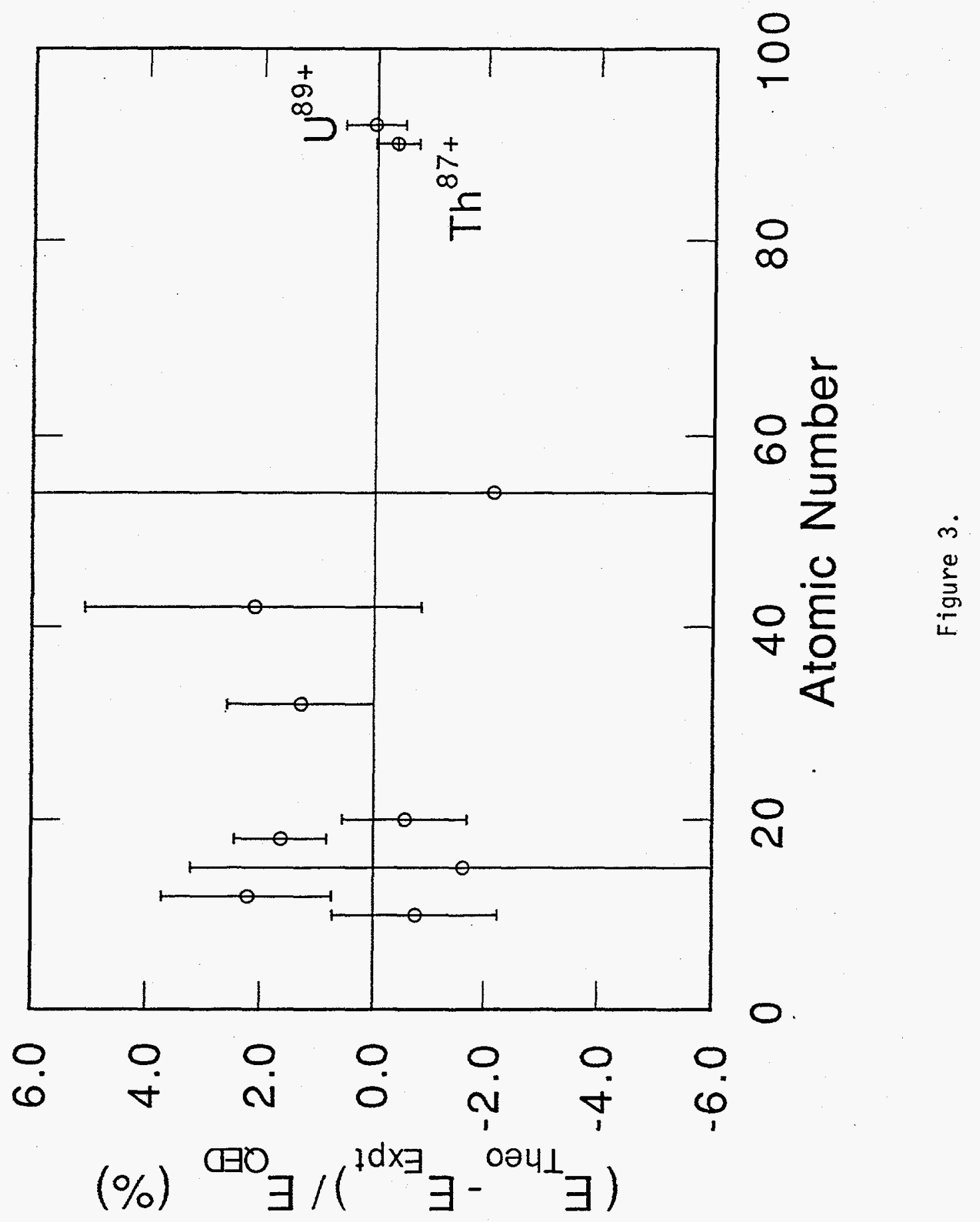



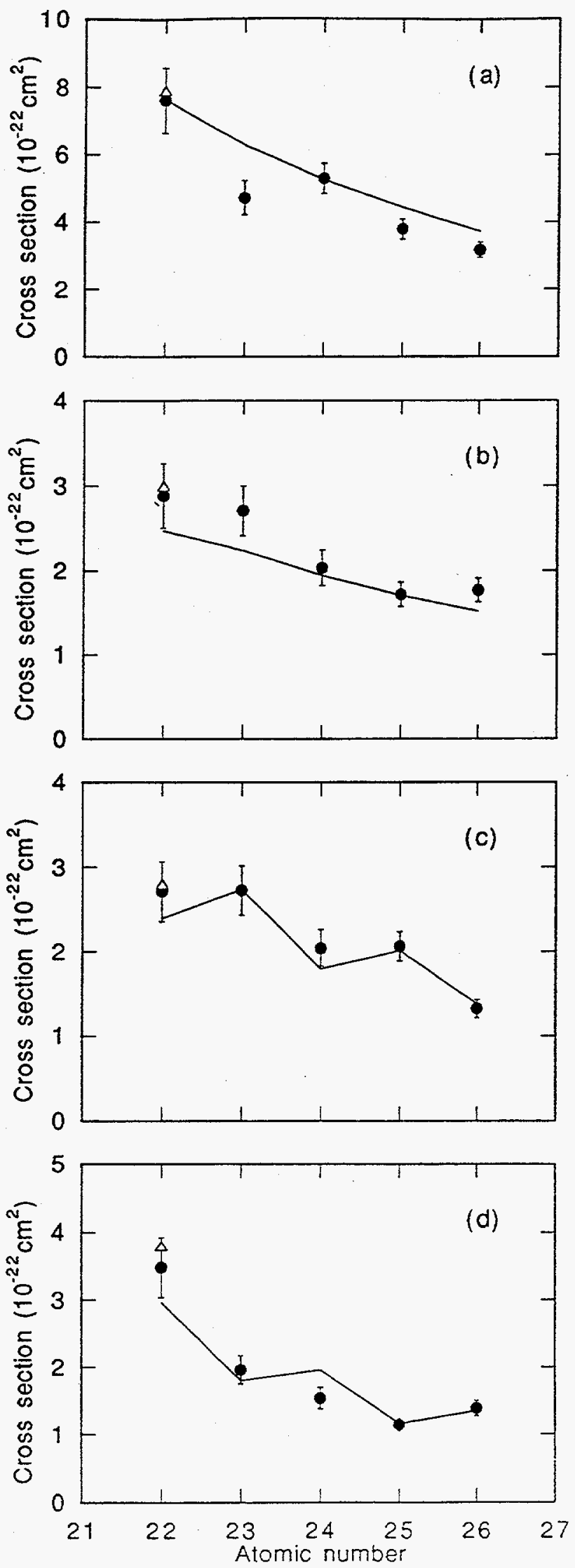

Figure 4. 

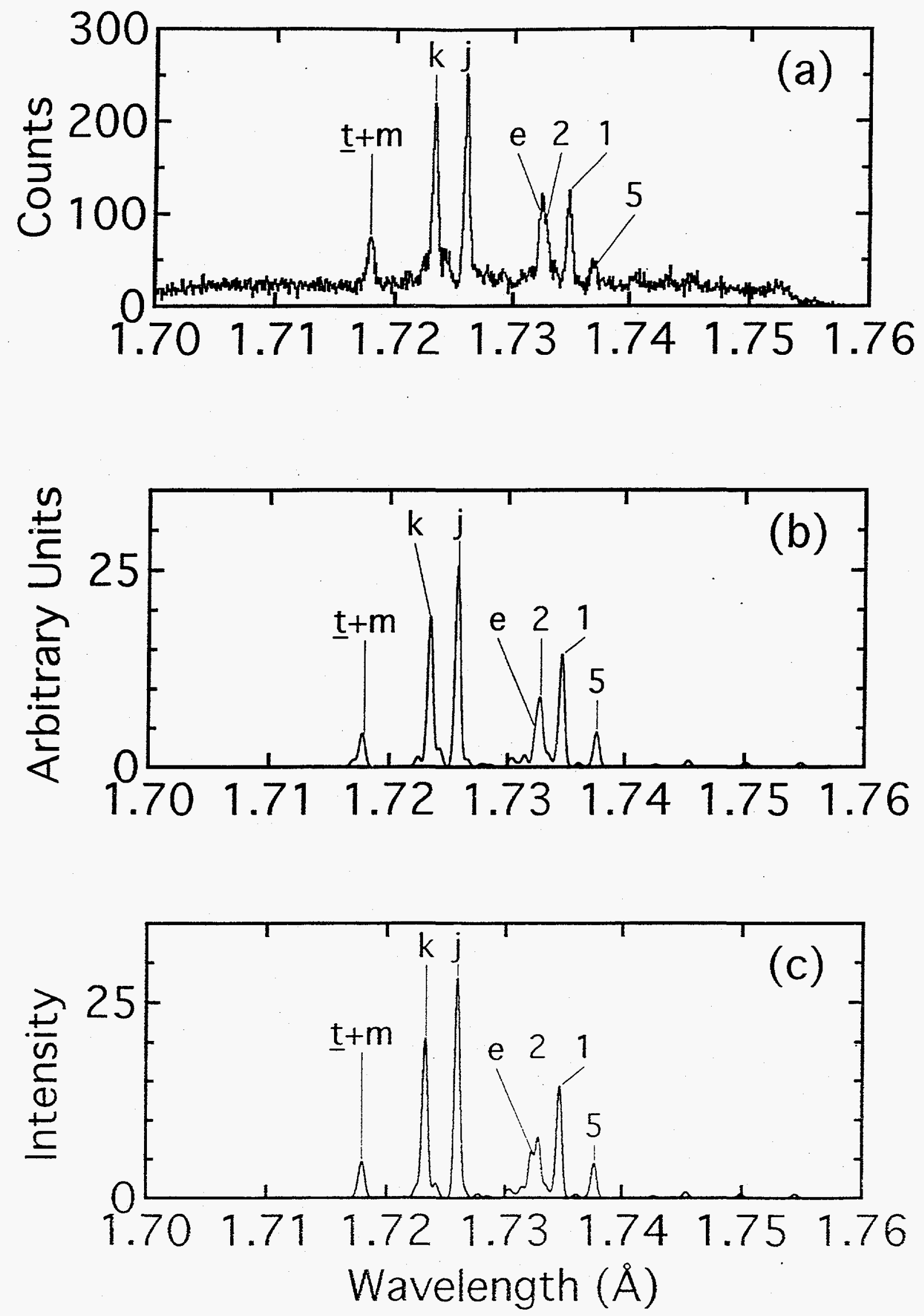

Figure 5. 


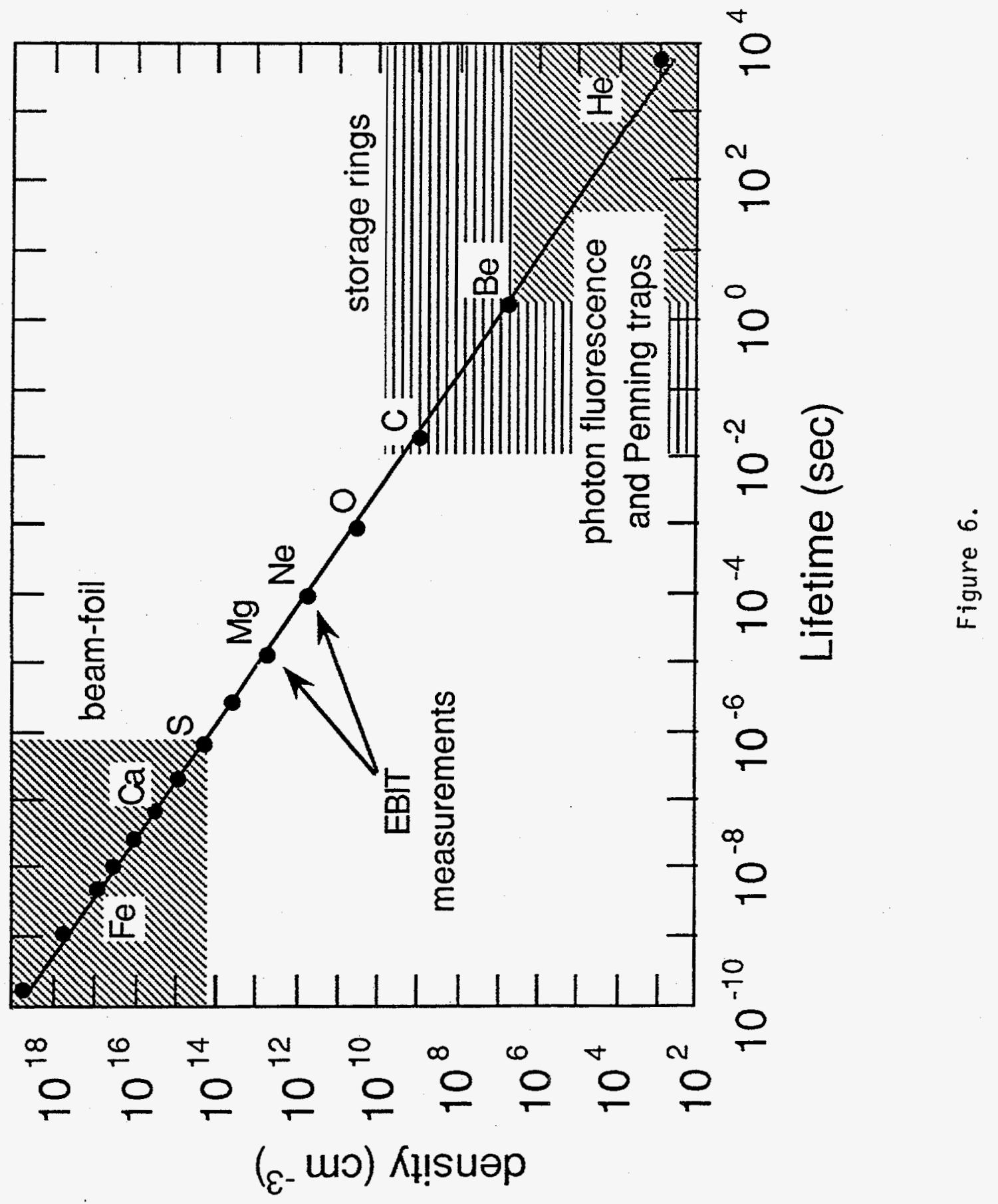



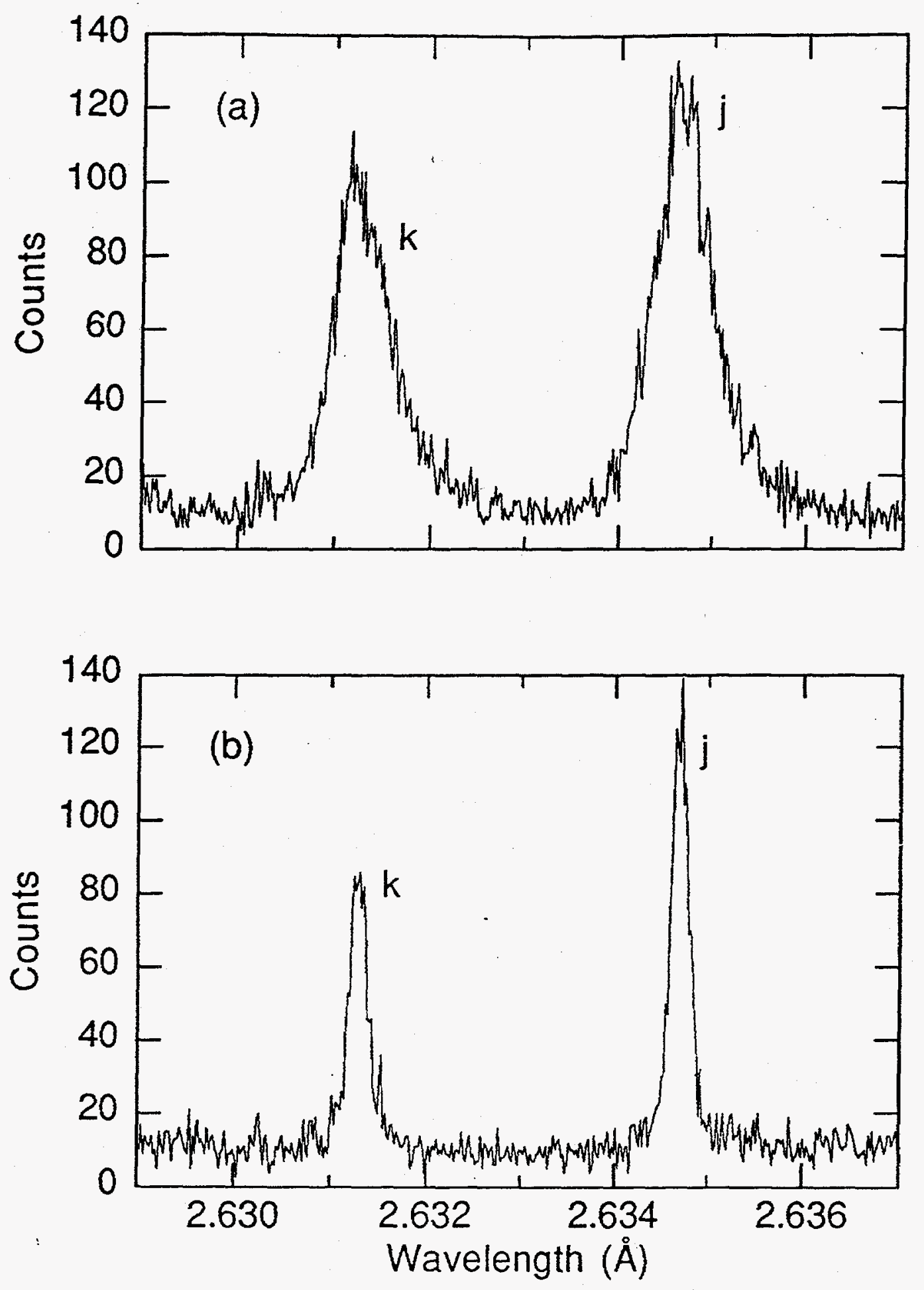

Figure 7. 